\title{
Elevated FAM3C promotes cell epithelial- mesenchymal transition and cell migration in gastric cancer
}

This article was published in the following Dove Press journal:

OncoTargets and Therapy

\section{Mengyue $\mathrm{Shi}^{1}{ }^{1 *}$ \\ Guihua Duan ${ }^{2, *}$ \\ Shuang Nie' \\ Shanshan Shen' \\ Xiaoping Zou'}

'Department of Gastroenterology, Drum Tower Hospital, Medical School of Nanjing University, Nanjing 210008, China; 'Department of Gastroenterology, First People's Hospital of Yunnan Province, Kunming University of Science and Technology, Kunming 650032, China

*These authors contributed equally to this work
Correspondence: Xiaoping Zou; Shanshan Shen

Department of Gastroenterology, Drum Tower Hospital, Medical School of Nanjing University, No. 32I Zhongshan Road, Nanjing 210008, China

Tel +86258310 6666;

+86 I3584049527

Fax +862583307115

Email zouxiaoping795@hotmail.com; shanshanshen33@hotmail.com
Background: Tumor metastasis is an important factor in treatment failure for advanced gastric cancer. Family with sequence similarity 3 member C (FAM3C) is known to play a critical role in inducing epithelial-mesenchymal transition in several cancer types, while its role in gastric cancer is unidentified. The aim of this study was to investigate the role of FAM3C in gastric cancer and provide new information on the receptor tyrosine-kinase pathway and cytokine-based therapies. Methods: FAM3C expression was tested in human gastric cancer tissue and adjacent normal mucosa, and the prognostic effect of FAM3C was analyzed in data from the Cancer Genome Atlas (TCGA). The role of FAM3C in gastric cancer proliferation and metastasis was investigated in vitro and in vivo. Western blot analysis and immunofluorescence were used to detect the underlying mechanisms.

Results: FAM3C expression was increased in gastric cancer tissue and showed cytoplasmic distribution. Gastric cancer patients with FAM3C overexpression had significantly worse prognoses based on TCGA data. In the gastric cancer cell lines MKN45 and AGS, knockdown of FAM3C dramatically attenuated cell migration, but had almost no influence on proliferation, while exogenous FAM3C promoted cell migration in a cell line with low FAM3C expression. Kyoto Encyclopedia of Genes and Genomes (KEGG) pathway enrichment of TCGA data showed that FAM3C was mainly associated with genes involved in focal adhesion, extracellular matrix-receptor interactions and the PI3K-Akt signaling pathway. Knockdown of FAM $3 C$ in gastric cancer cell lines significantly suppressed epithelial-mesenchymal transition, as demonstrated by increased expression of E-cadherin and decreased expression of Snail and Slug. Furthermore, knockdown of FAM $3 C$ strongly suppressed activation of the PI3K-Akt signaling pathway. Finally, we confirmed that $F A M 3 C$ knockdown significantly decreased metastatic lesions in vivo.

Conclusion: Our study demonstrated that FAM3C can promote gastric cancer metastasis both in vitro and in vivo. FAM3C should be taken into consideration for gastric cancer treatments involving inhibition of the ligands and downstream pathways of receptor tyrosine kinases.

Keywords: FAM3C, EMT, gastric cancer, metastasis

\section{Introduction}

Gastric cancer is one of the leading causes of cancer-related death worldwide. Treatment failure is often caused by inoperable disease at diagnosis or recurrent disease after resection. ${ }^{1}$ Preventing metastasis has always been a key objective of gastric cancer therapy. In metastatic disease, outcomes are poor, with median survival of $\sim 1$ year. ${ }^{1}$ A large proportion of gastric cancers are driven by receptor tyrosine kinases (RTKs). Although several RTK inhibitors and monoclonal antibodies have been introduced in 
gastric cancer therapy, ${ }^{1,2}$ therapeutic efficacy is attenuated by regulation of multiple cytokines and complicated RTK activation. ${ }^{3}$ Therefore, the identification of novel molecular targets for the development of more effective treatment methods is urgently needed.

Family with sequence similarity 3 member C (FAM3C) belongs to a cytokine-like gene family discovered by $\mathrm{Zhu}$ et al. ${ }^{4}$ This family includes FAM3A, FAM3B, FAM3C, and FAM3D, among which amino-acid-sequence comparison indicates an overall $31.6 \%-53.3 \%$ homology; however, these proteins have no homology with any known cytokines. ${ }^{4}$ FAM3C was first found to be involved in epithelial-mesenchymal transition (EMT) and metastasis in the mouse mammary epithelial cell line EpH4. ${ }^{5}$ FAM3C has also been found to participate in several cancer types. Strong cytoplasmic FAM3C expression in human breast cancer correlates with increased metastasis and decreased overall survival. ${ }^{5}$ Cytoplasmic overexpression of FAM3C detected by immunohistochemistry (IHC) is accompanied by downregulation of E-cadherin and positive expression of vimentin in human colorectal cancer tissue. FAM3C expression has also been found to be associated with TNM stage, EMT phenotype, and overall survival in colorectal cancer. ${ }^{6}$ FAM3C mRNA expression is markedly upregulated in tumor tissue and significantly associated with TNM stage and overall survival in esophageal squamous-cell carcinoma. ${ }^{7}$ The mechanism of FAM3C function has also been explored. In the renal tubular cell line HK2, FAM3C can independently induce EMT via the Akt-signaling pathway. Knockdown of FAM3C enhances the expression of E-cadherin and suppresses the phosphorylation of Akt. ${ }^{8} \mathrm{FAM} 3 \mathrm{C}$ overexpression has been reported to induce Akt phosphorylation in HepG2 cells. ${ }^{9}$ However, the role of FAM3C in gastric cancer remains unknown.

In this study, we investigated the role of FAM3C in gastric cancer metastasis. We discovered that FAM3C was overexpressed in gastric cancer and that overexpression of FAM3C was associated with significantly worse prognosis. Reduced FAM3C expression inhibited the metastasis of gastric cancer. Our results indicated that FAM3C was a culprit in promoting the metastasis of gastric cancer and might be a potential target for the development of anti-gastric cancer therapeutics.

\section{Methods}

\section{Patients and tissue samples}

Four pairs of pathologically confirmed gastric adenocarcinoma and adjacent noncancerous mucosal tissue samples were used for IHC and Western blot analysis. Patients were diagnosed with gastric cancer and underwent gastrectomy without preoperative systemic chemotherapy between January 2018 and March 2018 at the Nanjing Drum Tower Hospital. Before samples were collected, written informed consent from patients and approval from the institutional ethics committee of the Nanjing Drum Tower Hospital were obtained.

\section{Cell culture}

The human gastric cancer cell lines MKN45, AGS, HGC27, NCI-N87, SNU601, and SNU-791 and the immortalized normal human gastric epithelial cell line GES1 were maintained in our laboratory or purchased from the Type Culture Collection of the Chinese Academy of Sciences, Shanghai, China. All cell lines were cultured in RPMI 1640 medium (Thermo Fisher Scientific, Waltham, MA, USA) supplemented with 10\% FBS (Biological Industries, Beit-Haemek, Israel) and $1 \%$ penicillin-streptomycin $(50 \mathrm{U} / \mathrm{mL}, 50 \mu \mathrm{g} / \mathrm{mL}$; Thermo Fisher Scientific) under $5 \% \mathrm{CO}_{2}$.

\section{shRNA-knockdown assay}

FAM $3 C$ shRNAs or scrambled control shRNA were constructed into an hU6-MCS-ubiquitin-EGFP-internal ribosome-entry site-puromycin lentiviral vector and packaged into lentivirus particles in $293 \mathrm{~T}$ cells (GeneChem, Shanghai, China). AGS and MKN45 cells were plated into six-well plates at concentrations of $2 \times 10^{5}$ and $3 \times 10^{5}$ cells per well. The culture medium was replaced with complete culture medium including $1 \mu \mathrm{g} / \mathrm{mL}$ Polybrene (GeneChem) immediately before adding the lentiviral particles. The multiplicity of infection value was 50 for each cell line. Three days after infection, cells were selected with $1 \mu \mathrm{g} / \mathrm{mL}$ puromycin (R\&D Systems, Minneapolis, MN, USA) to generate stable FAM3C-knockdown cells. Transfection efficiency was monitored based on GFP-expression rate under fluorescence microscopy (CellSens; Olympus, Tokyo, Japan). FAM3Cknockdown efficiency was determined by quantitative reverse transcription (qRT) PCR and Western blot analysis of mRNA and protein levels, respectively. Two shRNAs targeting FAM $3 C$ were used, the sequences of which were: shFAM3C1, 5'-CATACAAGATGGAACAATA-3'; and shFAM3C2, 5'-TGCAAGTTTAGGAAATCTA-3'.

\section{Western blot analysis}

For protein extraction from cultured cells, cells were washed twice with cold PBS and lysed in RIPA buffer (Beyotime, Haimen, China) containing an EDTA-free protease-inhibitor cocktail (Hoffman-La Roche, Basel, Switzerland) on ice for 15 minutes. Tissue was also lysed in RIPA buffer (Beyotime) containing an EDTA-free protease-inhibitor cocktail 
(Hoffman-La Roche) in an ultrasonic tissue homogenizer (Scientz Biotechnology, Ningbo, China) for 5 minutes. Lysates were centrifuged for 15 minutes $(12,000 \mathrm{rpm}$, $4^{\circ} \mathrm{C}$ ). The supernatant was collected and protein concentrations determined using bicinchoninic acid (BCA) assay kit (Beyotime). Equal amounts of protein were separated with $8 \%-12 \%$ SDS-PAGE and then electrophoretically transferred onto a polyvinylidene difluoride membrane (Millipore, Billerica, MA, USA). After being blocked with $5 \%$ nonfat milk in Tris-buffered saline containing $0.1 \%$ Tween 20 for 2 hours, membranes were incubated with specific primary antibodies according to the manufacturer's instructions overnight at $4^{\circ} \mathrm{C}$, followed by incubation with appropriate horseradish peroxidase-conjugated secondary antibodies for 2 hours. Signals generated by enhanced chemiluminescence (Millipore) were recorded with a 4600 imaging system (Tanon, Shanghai, China). Primary and secondary antibodies included those against FAM3C (HPA050548, Sigma-Aldrich, St Louis, MO, USA), E-cadherin (CST, 3195), vimentin (CST, 5741), p-Akt (Ser473; CST, 4060), Akt (CST, 2920), p-GSK3ß (Ser9; CST, 5558), GSK3 $\beta$ (CST, 12456), Snail (CST, 3897), Slug (CST, 9585), and $\beta$-actin (A5441; Sigma-Aldrich).

\section{RNA extraction and qRT-PCR}

Total RNA was isolated from cells with RNAiso Plus Reagent (Takara, Kusatsu, Japan). RT was performed with PrimeScript RT master mix (Takara) according to the manufacturer's instructions. Quantification of gene expression was performed using an SYBR Premix Ex Taq II kit (Takara) on a LightCycler 96 system (Roche). Gene expression was normalized to the expression of $A C T B$. All reactions were performed in triplicate. The primer sequences used were: $F A M 3 C$, forward 5'-GCAAAGTTGGTGGTAGCTGTG-3', reverse 5'-AGCAAAATGCTTCTCAGGGC-3'; and $A C T B$, forward 5'-CTACGTCGCCCTGGACTTCGAGC-3', reverse 5'-GATGGAGCCGCCGATCCACACGG-3'.

\section{IHC}

IHC was carried out as described previously. ${ }^{10}$ Briefly, following endogenous peroxidase neutralization and antigen retrieval by microwave irradiation, slides were preincubated with blocking serum and incubated overnight with the FAM3C antibody (HPA050548). All sections were evaluated by two independent pathologists. To assess FAM3C expression, five views per slide were chosen randomly and at least 1,000 cells counted in each view. Scores were assigned to each sample based on FAM3C-staining intensity ( 0 , negative; 1 , weak; 2 , medium; 3 , strong) and on the percentage of FAM3C-positive cells (1) $0-25 \% ; 20$ 26\%-50\%; $3051 \%-75 \%$; and $40>75 \%$ ). A tumor-staining index was calculated for each sample by multiplying the staining-intensity score by the percentage score.

\section{Cell-proliferation analysis}

AGS, MKN45, and HGC27 cells were plated into 96-well plates at a concentration of $3 \times 10^{3}$ cells per well in $100 \mu \mathrm{L}$ complete growth medium. FAM3C $(0,10$, or $100 \mathrm{ng} / \mathrm{mL})$ was added to the HGC27 cells (Sino Biological, Beijing, China) 24 hours after seeding. Cell viability was analyzed 1, 2, 3 and 4 days after cell seeding with Cell Counting Kit-8 (Dojindo, Kumamoto, Japan) according to the manufacturer's instructions.

\section{Wound-healing assay}

AGS cells transfected with scrambled control shRNA, FAM3C shRNAs, or HGC27 cells were plated into sixwell plates at a concentration of $6 \times 10^{5}$ cells per well. Cells reached $\sim 90 \%$ confluence 24 hours after cell seeding. The cell monolayer was scratched with a yellow $200 \mu \mathrm{L}$ pipette tip to generate a linear scratch wound. The culture medium was replaced with FBS-free RPMI 1640 medium. FAM3C $(0,10$, or $100 \mathrm{ng} / \mathrm{mL})$ was added to the HGC27 cells. Cells were cultured for 24 hours. Scratch-wound images at 0 and 24 hours were captured using an Olympus IX71 microscope, and wound-healing ability was calculated based on the relative cell-free area normalized to that in the 0 -hour image.

\section{Transwell assay}

The migration ability of HGC27 cells treated with FAM3C and AGS cells transfected with scrambled control shRNA or FAM $3 C$ shRNAs was tested in a transwell Boyden chamber $(8 \mathrm{~mm})$. Cells were harvested and suspended in FBS-free RPMI 1640 culture medium at a concentration of $10^{5}$ cells $/ \mathrm{mL}$. RPMI 1640 medium $(0.8 \mathrm{~mL})$ containing $20 \%$ FBS was added to the lower compartment. Then, 0.5 $\mathrm{mL}$ cell suspension was added to the upper chamber. Transwell-containing plates were incubated for $\sim 24$ hours in a $5 \%$ $\mathrm{CO}_{2}$ atmosphere saturated with $\mathrm{H}_{2} \mathrm{O}$. Cells passing through the filter membrane were fixed with $4 \%$ paraformaldehyde at room temperature for 15 minutes, washed three times with distilled water, and stained with $0.5 \%$ crystal violet. We then gently removed the cells remaining on the upper surface of the filter membrane with a cotton swab. Images of the lower surfaces were captured using microscopy (five fields per chamber; CX31; Olympus) and the number of cells per field counted and analyzed. 


\section{Colony-formation assay}

Cells were plated in six-well plates in $2 \mathrm{~mL}$ complete medium. Numbers of negative-control and FAM $3 C$-knockdown cells per well were 3,000 for AGS cells and 1,000 for MKN45 cells. Complete medium $(1 \mathrm{~mL})$ was added 4 days after cell seeding. After 10-14 days, colonies were fixed in methanol and stained with $0.5 \%$ crystal violet. The number of colonies was calculated in ImageJ.

\section{Immunofluorescence}

Cells from each group were seeded in 24-well plates at a density of $3 \times 10^{4}$ cells per well. At 24 hours after seeding, cells were fixed with $4 \%$ paraformaldehyde for 15 minutes at room temperature and permeabilized with $0.5 \%$ Triton X-100 for 30 minutes. After blocking with 5\% BSA for 1 hour at room temperature, cells were incubated with E-cadherin (1:100), FAM3C (1:100), or vimentin (1:200) antibodies overnight at $4^{\circ} \mathrm{C}$. Cells were washed 3 times and then incubated with Dylight649 goat antirabbit IgG (1:200, 111-495-003; Jackson ImmunoResearch, West Grove, PA, USA) for 1 hour at room temperature in the dark. Then, cells were incubated with DAPI (1:50; KeyGen Biotech, Nanjing, China) for 5 minutes. Cells were visualized using the CellSens.

\section{Xenograft experiments}

Six-week-old male BALB/c nu/nu mice were purchased from Vital River Laboratories. For lung-metastasis xenografts, control MKN45 cells and stable FAM3C-knockdown MKN45 cells were generated as described earlier: $2 \times 10^{6}$ cells were suspended in $200 \mu \mathrm{L}$ cold PBS and injected into the lateral tail vein of five mice in each group. Four weeks after cell implantation, the mice were killed. Then, lungs were fixed with $4 \%$ paraformaldehyde and analyzed with H\&E staining. Numbers of metastatic lesions on the largest section of each sample were calculated and analyzed. All xenograft experiments were approved by the Ethics Review Committee for Animal Experimentation at Drum Tower Hospital (Nanjing, China). All animal procedures were performed in compliance with the guidelines set by the Animal Care Committee, and all efforts were made to minimize potential pain and discomfort in the animals.

\section{The Cancer Genome Atlas (TCGA) TCGA data analysis and Kyoto Encyclopedia of Genes and Genomes (KEGG) pathway enrichment}

Gene-expression and survival data for TCGA gastric adenocarcinoma (provisional) patients $(\mathrm{n}=478)$ were downloaded from the cBioPortal website (http://www.cbioportal.org).
The cutoff value for FAM3C mRNA expression (RNA Seq V2 RSEM) was 2,500, and survival analysis was performed using the Kaplan-Meier method. $P$-values were calculated with a log-rank test. Genes positively related to $F A M 3 C$ in gastric adenocarcinoma (provisional) data from TCGA with Pearson score $>0.3$ were collected and underwent KEGG pathway enrichment using the DAVID online analysis tool (https://david.ncifcrf.gov) and Cytoscape following the instructions. ${ }^{11}$

\section{Statistics}

All experiments were performed in triplicate unless otherwise indicated. Data are presented as means $\pm \mathrm{SD}$. Differences in mRNA expression, colony number, cell-free area, cell numbers, metastasis-lesion number, and IHC protein expression between or among groups were evaluated by ANOVA, Student's $t$-test, or nonparametric test. Differences were considered significant if $P<0.05$. All statistical analyses were performed using Prism 6.0.

\section{Results \\ FAM3C overexpression was associated with worse prognosis in gastric cancer patients}

To investigate whether FAM3C also participates in gastric cancer malignancy, we first detected the protein level of FAM3C in human gastric cancer tissue and adjacent normal gastric mucosa using Western blot analysis. The FAM3C protein level was increased in gastric cancer tissue. As FAM3C was found to be primarily an EMT inducer in several other cancers, ${ }^{5}$ we further detected the epithelial cell markers E-cadherin and vimentin. Decreased E-cadherin protein levels and increased vimentin expression were related to increased FAM3C levels in three of four gastric cancer tissue samples compared to those in adjacent normal gastric mucosa (Figure 1A).

We also detected FAM3C expression in gastric cancer tissue and adjacent noncancerous mucosa by IHC. Based on calculated IHC scores, FAM3C expression in cancer and noncancerous tissue was not significantly different (Figure 1B). Interestingly, FAM3C expression showed cytoplasmic distribution in gastric cancer tissue, but showed granular distribution in normal mucosa (Figure 1C). It has been reported that cytoplasmic FAM3C, which represents functional protein that is ready for secretion, ${ }^{5}$ is related to worse prognosis in colorectal cancer types. ${ }^{6}$

We then evaluated the prognostic value of FAM3C expression in gastric cancer based on the stomach-adenocarcinoma 
A

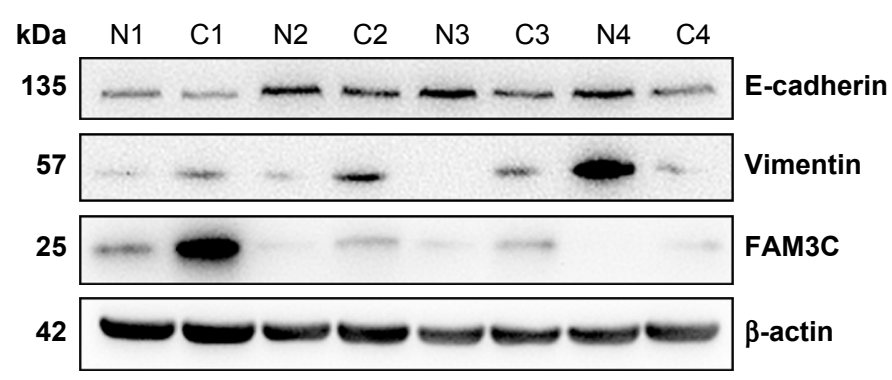

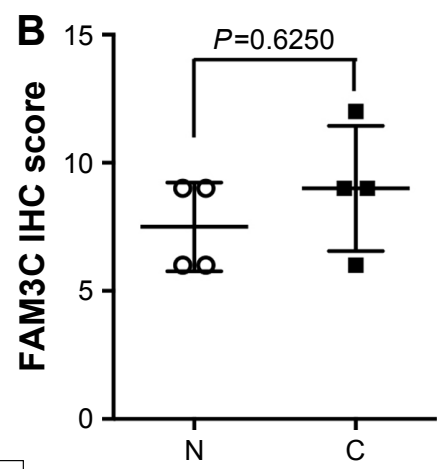
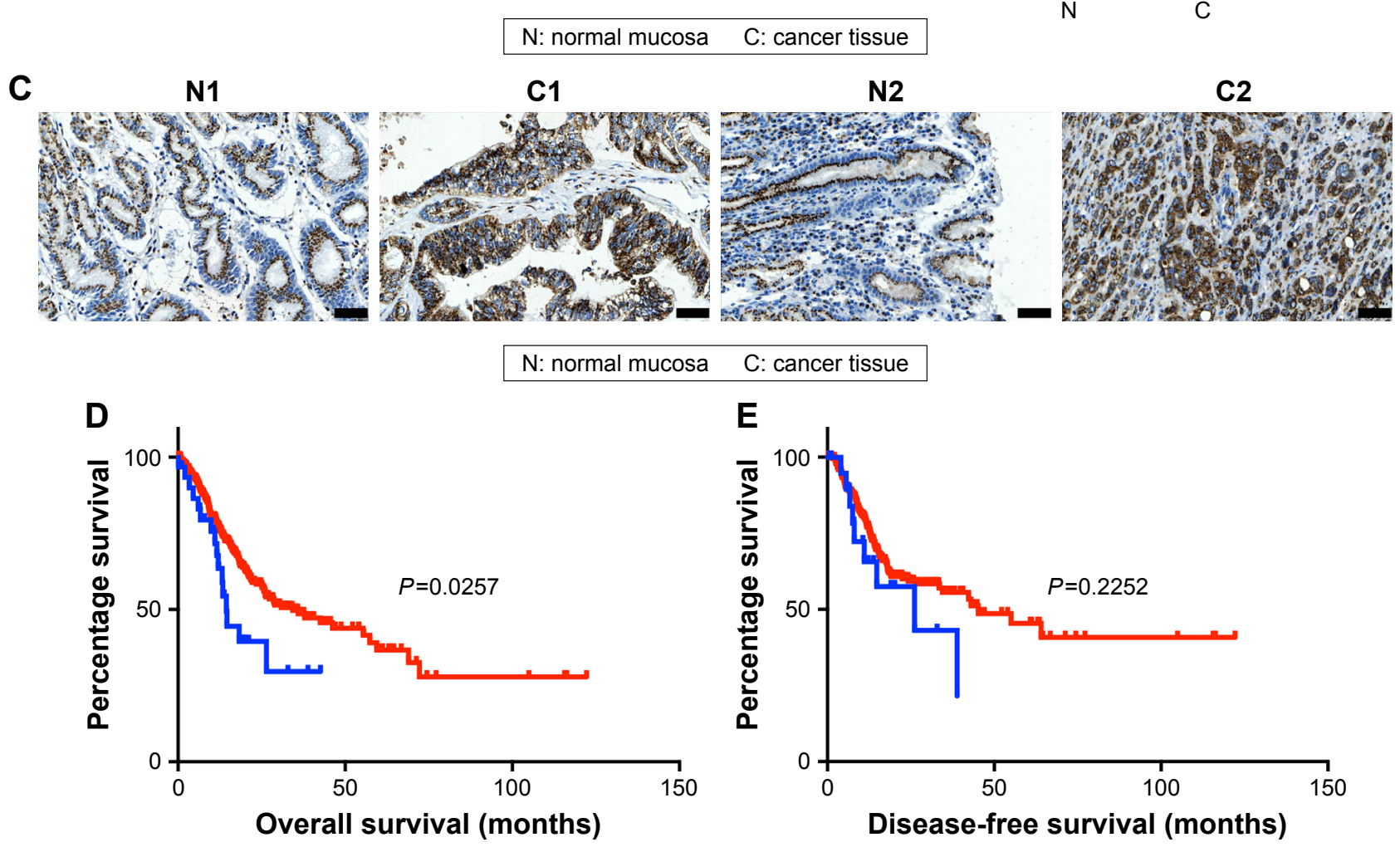

E

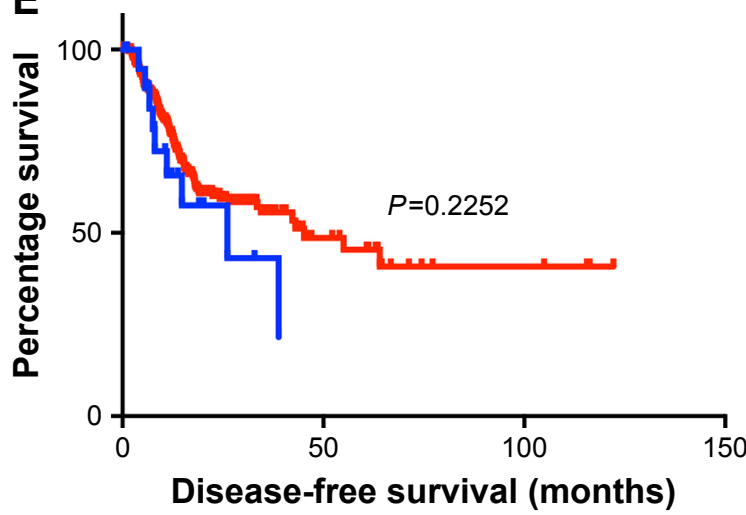

-FAM3C high $\leftarrow$ FAM3C low

Figure I FAM 3 C was overexpressed in gastric cancer tissue, and high FAM3C expression was related to poor overall survival.

Notes: (A) The protein level of FAM3C was increased in gastric cancer tissue compared to adjacent normal gastric tissue. Increased FAM3C was related to decreased levels of E-cadherin in three of four pairs of gastric cancer tissue than adjacent normal gastric mucosa. Vimentin expression was lower in three of four gastric cancer tissue samples than adjacent normal mucosa. (B) Statistics of the FAM3C IHC scores in four pairs of gastric cancer and adjacent noncancerous tissue. (C) Typical images of FAM3C IHC staining. FAM3C expression showed granular distribution in normal mucosa, but cytoplasmic distribution in cancer tissue. Bar $50 \mu$ m. (D) FAM3C overexpression was significantly associated with poor overall survival. Median overall survival time was 34.26 months in the low-FAM3C-expression group and I4.42 months in the high-FAM3Cexpression group $(P=0.0257)$. (E) Median disease-free survival time was 45.2 months in the low-FAM3C-expression group and 26.12 months in the high-FAM3C-expression group $(P=0.4007)$.

Abbreviation: IHC, immunohistochemistry.

database of TCGA $(\mathrm{n}=478)$. The median overall survival time was 34.26 months in the low-FAM3C-expression group and 14.42 months in the high-FAM3C-expression group $(P=0.0257$; Figure 1D). The median disease-free survival time in the low- and high-FAM3C-expression groups was 45.2 months and 26.12 months respectively $(P=0.4007$; Figure 1E). These data showed that FAM3C participated in gastric cancer malignance and that overexpression of FAM3C in gastric cancer was associated with shorter overall survival.

\section{Knockdown of FAM3C suppressed gastric cancer-cell migration in vitro}

We further studied the role of FAM3C in gastric cancer cell lines. First, we detected mRNA and protein levels of FAM3C in six human gastric cancer cell lines and the immortalized human gastric epithelial cell line GES1. Most of the gastric cancer cell lines showed higher FAM3C expression than GES1 cells at both mRNA and protein levels (Figure 2A and B), among which MKN45 had the highest FAM3C 
A

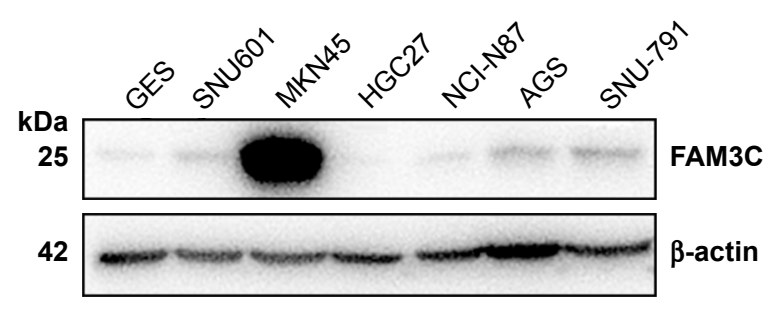

B

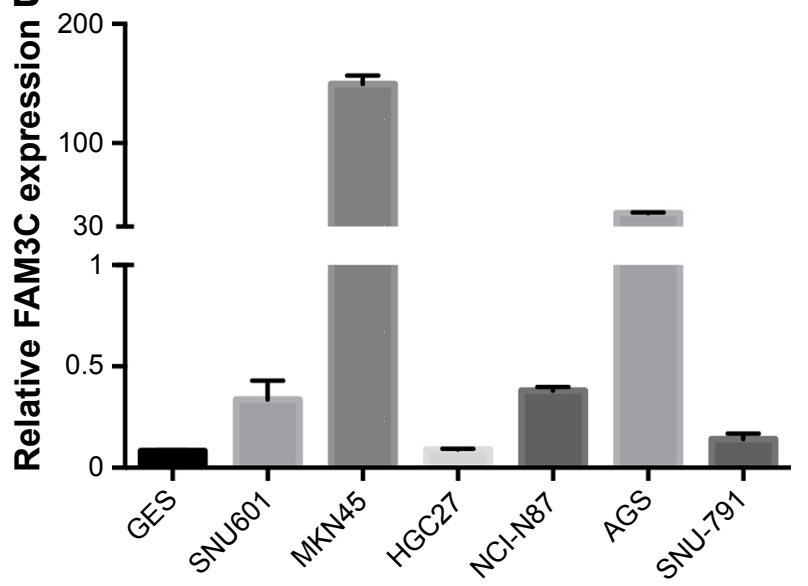

C

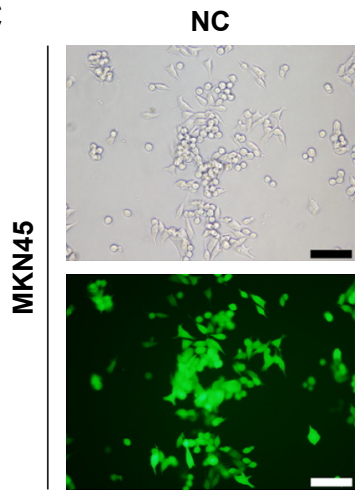

shFAM3C1

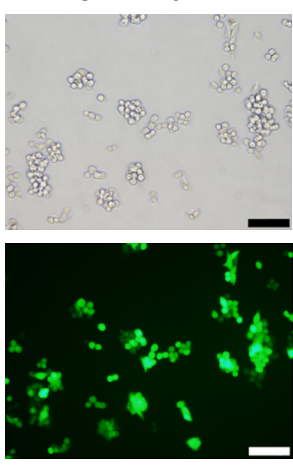

ShFAM3C2
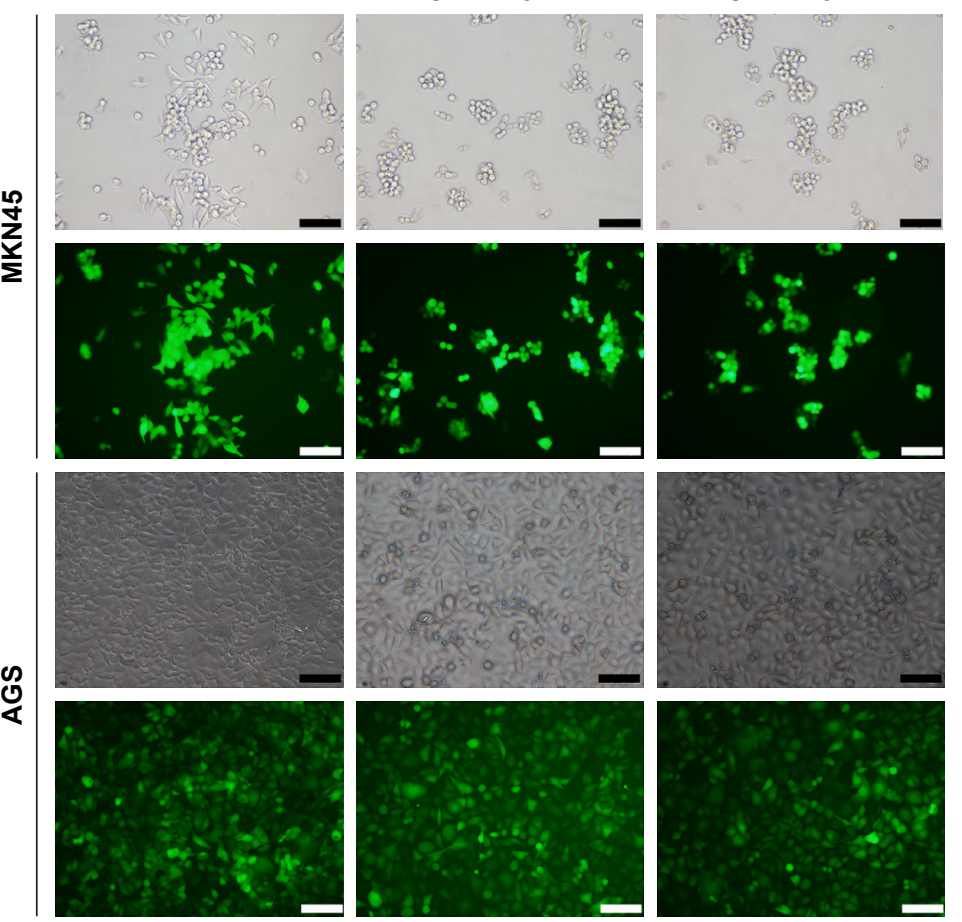

E

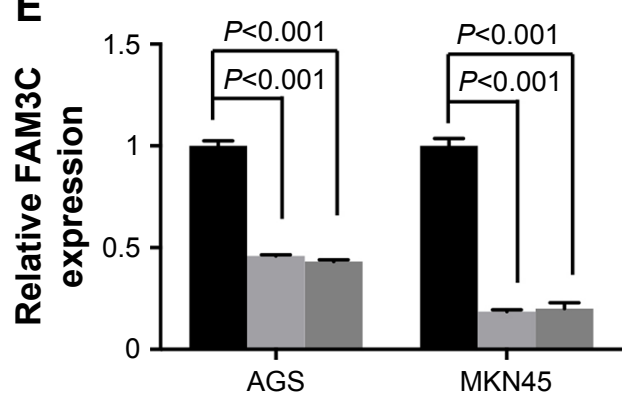

NC $\quad$ shFAM3C1 $\quad$ shFAM3C2

Figure 2 Knockdown of $F A M 3 C$ in gastric cancer cell lines by shRNA.

Notes: FAM3C expression was detected in an immortalized human gastric epithelial cell line, GESI, and in six human gastric cancer cell lines at the protein (A) and mRNA (B) levels. (C) Transfection efficiency of the lentiviral vector expressing shRNAs targeting FAM3C. Bar $100 \mu$ m. Two shRNAs achieved efficient knockdown of FAM3C at the protein (D) and mRNA levels (E).

Abbreviation: NC, negative control. 
expression. The mRNA level of FAM3C in MKN45 cells was 150 -fold higher than the average expression level of the seven cell lines. The cell line with the next-highest FAM3C expression was AGS, in which the mRNA level of FAM3C was 41-fold higher than the average level (Figure 2A and B). Therefore, we chose MKN45 and AGS cells for further knockdown assays.

To achieve stable knockdown of $F A M 3 C$, two shRNAs targeting FAM3C mRNA were constructed into a lentiviral vector expressing GFP and a puromycin-resistance gene. AGS and MKN45 cells were transfected with the two shRNAs and selected with $1 \mu \mathrm{g} / \mathrm{mL}$ puromycin. Transfection efficiency was examined based on the proportion of cells expressing GFP under fluorescence microscopy (Figure 2C), and knockdown efficiency was determined by Western blot analysis and qRT-PCR. Both shRNAs achieved satisfactory knockdown efficiency (Figure 2D and E) and could be used for further study.

As shown in CCK8 and colony-formation assays, decreased FAM3C in MKN45 and AGS cells did not influence cell proliferation (Figure $3 \mathrm{~A}-\mathrm{C}$ ). Considering that $\mathrm{FAM} 3 \mathrm{C}$ has been found to promote EMT in several cancer types, we further tested whether knockdown of FAM3C influenced gastric cancer-cell migration. MKN45 cells are semiadherent cells that cannot be used to conduct woundhealing or transwell assays. Therefore, we used AGS cells for these assays. Interestingly, knockdown of $F A M 3 C$ significantly influenced the wound-healing ability (Figure 3D and E) and migration (Figure $3 \mathrm{~F}$ and $\mathrm{G}$ ) of AGS cells.

\section{Knockdown of FAM3C attenuated EMT and suppressed activation of $\mathrm{PI} 3 \mathrm{~K}-\mathrm{Akt}$ signaling pathway}

FAM3C has also been referred to as an interleukin-like EMT inducer for its role in inducing cancer cells to undergo EMT in several cancer types. ${ }^{5,6,12} \mathrm{We}$ also found that overexpression of FAM3C was associated with decreased E-cadherin in human gastric cancer tissue (Figure 1A). Therefore, we tested whether knockdown of FAM 3 in gastric cancer could cause the upregulation of epithelial markers. As shown by the immunofluorescence results, knockdown of FAM3C resulted in increased levels of E-cadherin and decreased levels of vimentin, along with reduced mesenchymal cell morphology (Figure 4A). These findings were also validated by Western blot analysis (Figure 4C). As AGS cells bear mutated E-cadherin, Western blot analysis could not detect E-cadherin in these cells. ${ }^{13}$ To explore the underlying mechanism of FAM3C in gastric cancer, we exported the genes positively related to $F A M 3 C$ (Pearson score $\geq 0.3$; Table S1) in the gastric adenocarcinoma database of TCGA and conducted KEGG pathway enrichment (Table 1). We found that genes positively related to $F A M 3 C$ were concentrated mainly in the focal adhesion, extracellular matrix-receptor interaction and PI3K-Akt signaling-pathway categories ( $P<0.05$ and $>5 \%$ associated genes; Figure 4B). The PI3K-Akt-GSK3 $\beta$ pathway is a canonical pathway downstream of RTKs that participates in numerous intracellular molecular processes. Akt phosphorylated by PI3K is activated and phosphorylates GSK $3 \beta$ directly, which causes the inactivation of GSK $3 \beta$. Activation of GSK $3 \beta$ leads to the degradation of Snail, an EMT transcription factor. Therefore, inhibiting the PI3K-Akt pathway results in the activation of GSK $3 \beta$ and the subsequent degradation of Snail, which has been reported to attenuate EMT and suppress metastasis in lung cancer, breast cancer, gallbladder cancer, and gastric cancer. ${ }^{14-17}$ We detected activation of the PI3K-Akt signaling pathway using Western blot analysis. Knockdown of $F A M 3 C$ resulted in decreased levels of phosphorylated Akt and corresponding decreases in p-GSK3 $\beta$. Knockdown of FAM3C also caused downregulation of Snail and Slug, two EMT-related transcription factors. These data suggest that FAM3C might regulate EMT via the canonical EMTregulating PI3K-Akt-GSK3 $\beta$-Snail pathway.

\section{Exogenous FAM3C activated PI3K-Akt signaling pathway and promoted gastric cancer-cell migration in vitro}

To study whether exogenous FAM3C promoted gastric cancer-cell migration, we chose a gastric cancer cell line with low FAM3C expression, HGC27, and added exogenous FAM3C cytokine to the culture medium. As shown in the CCK8 assay, $100 \mathrm{ng} / \mathrm{mL}$ FAM3C slightly promoted cell proliferation after 48 hours, while $10 \mathrm{ng} / \mathrm{mL}$ FAM3C did not have this effect (Figure 5A). Interestingly, both $10 \mathrm{ng} / \mathrm{mL}$ and $100 \mathrm{ng} / \mathrm{mL}$ FAM3C promoted wound healing and cell migration (Figure 5B-E). Exogenous FAM3C also caused increased phosphorylation of Akt and GSK3 $\beta$, with a decrease in E-cadherin (Figure 5F).

\section{Decreased FAM3C levels attenuated cell metastasis in vivo}

To evaluate the potential role of FAM3C in gastric cancer metastasis further, a lung-metastasis xenograft mouse model of MKN45 cells was employed: $2 \times 10^{6}$ cells were injected into 
A

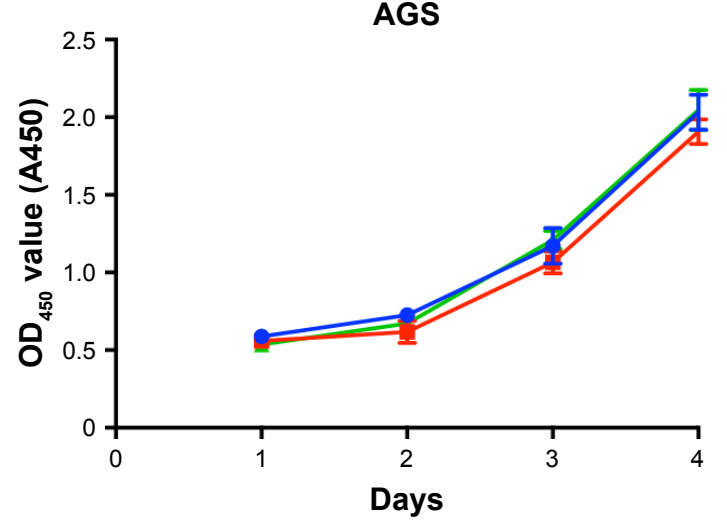

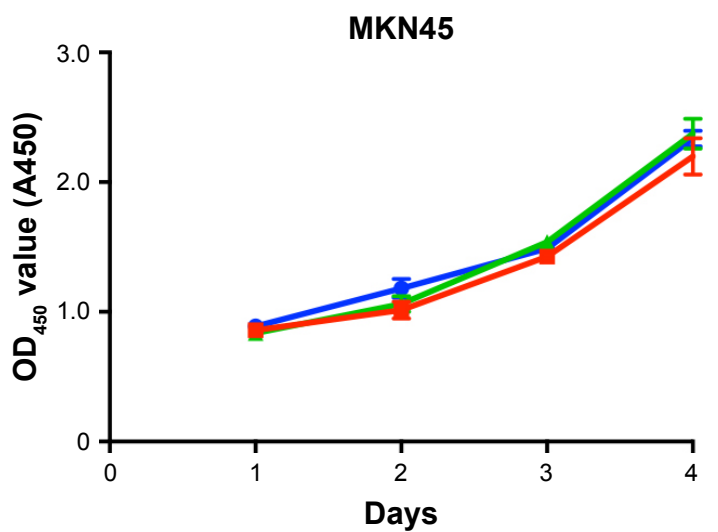

B

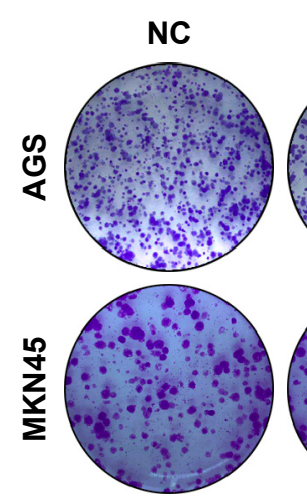

D
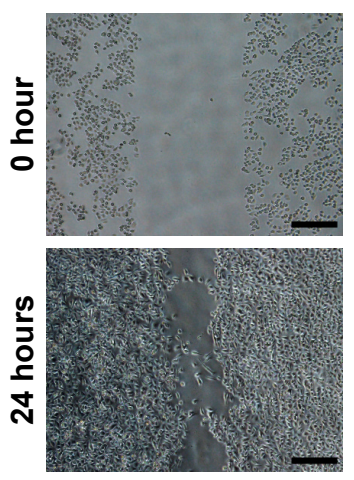

$\mathbf{F}$

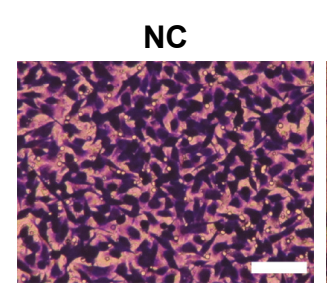

shFAM3C1
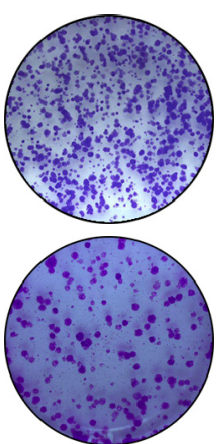

shFAM3C1
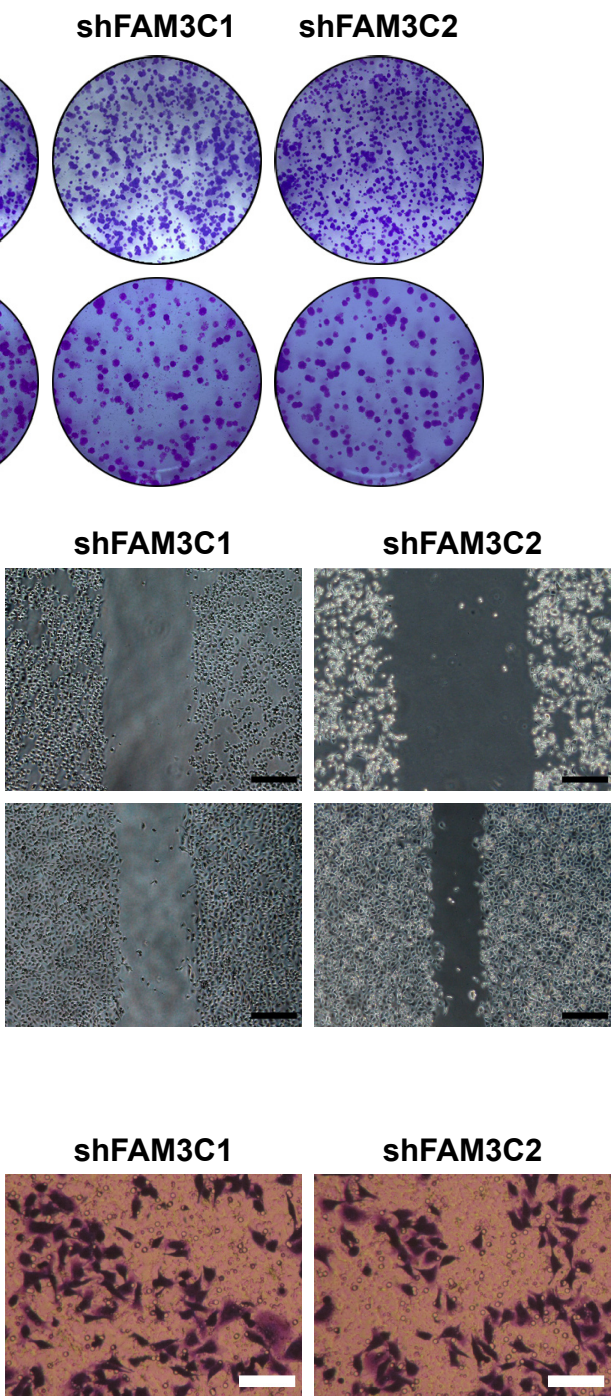
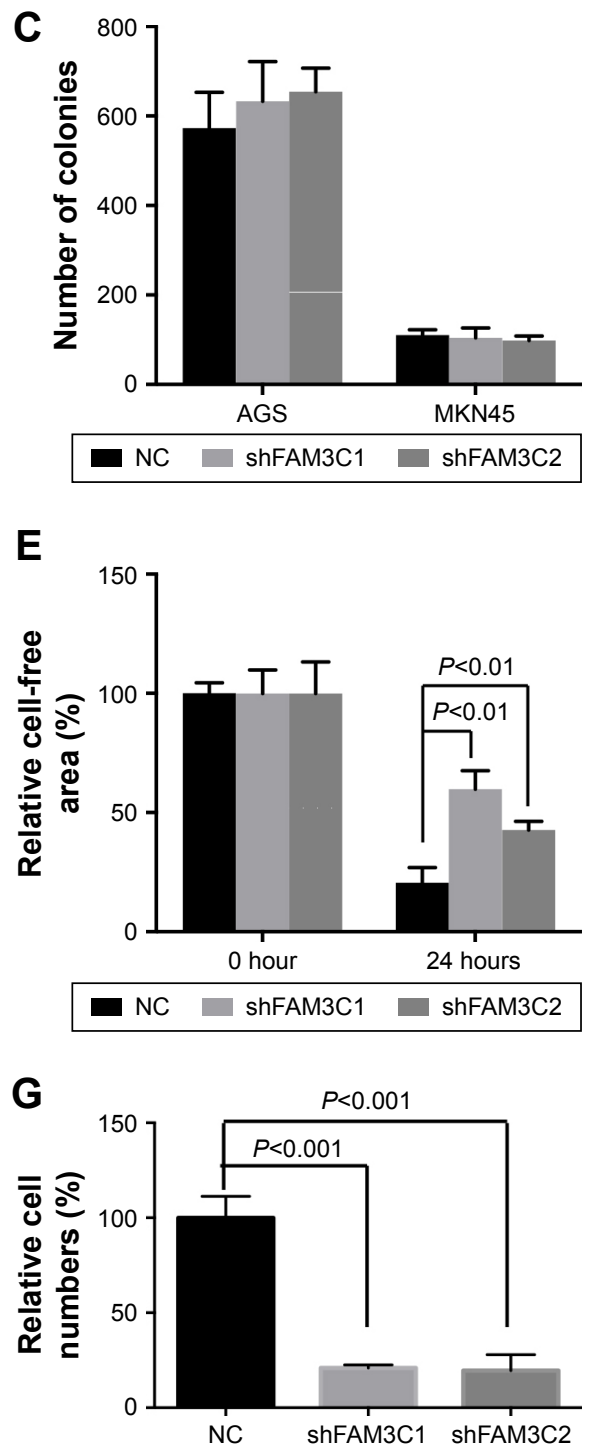

Figure 3 Knockdown of FAM3C did not influence cell proliferation, but did attenuate gastric cancer-cell migration in vitro.

Notes: $(\mathbf{A}) \mathrm{OD}_{450}$ values of control and FAM3C-knockdown cells at 24 hours, 48 hours, 72 hours, and 96 hours after the cells were seeded into 96 -well plates showed no differences in AGS or MKN45 cells. (B, C) The number of formed colonies was not significantly different between control and FAM3C-knockdown cells. Images (B) and statistics (C) regarding the number of colonies. (D, E) Knockdown of FAM3C in AGS cells resulted in decreased wound-healing area 24 hours after scratching. Representative images $($ bar $200 \mu \mathrm{m})(\mathbf{D})$ and statistics $(\mathbf{E})$ regarding the percentage of uncovered area. $(\mathbf{F}, \mathbf{G})$ Knockdown of FAM3C in AGS cells had reduced the number of migrated cells in the transwell assay at 24 hours after cell seeding. Representative images (bar $100 \mu \mathrm{m})(\mathbf{F})$ and statistics $(\mathbf{G})$ regarding migrated cell numbers.

Abbreviation: NC, negative control. 
A
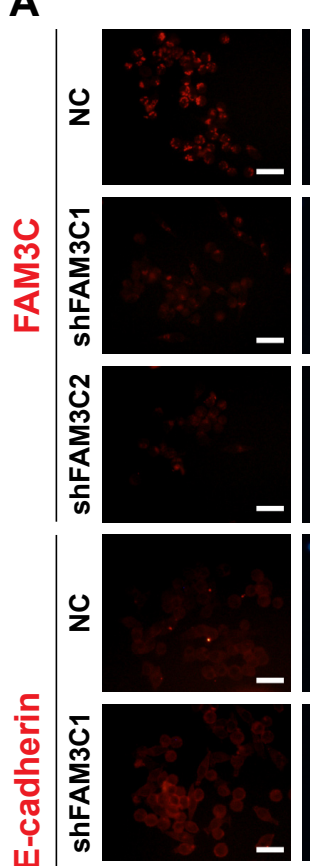

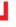

DAPI
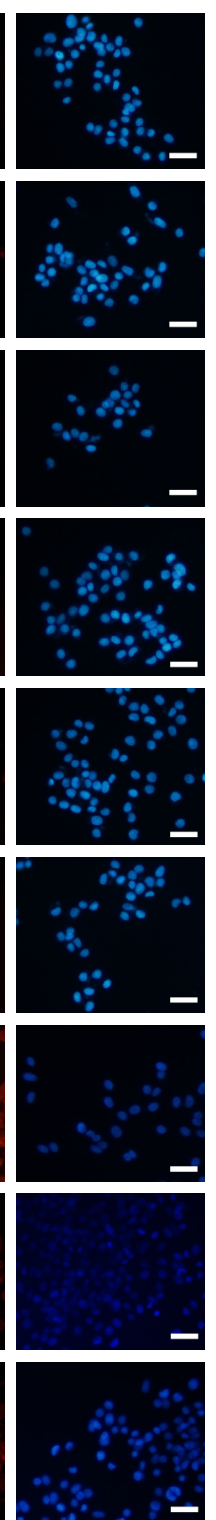
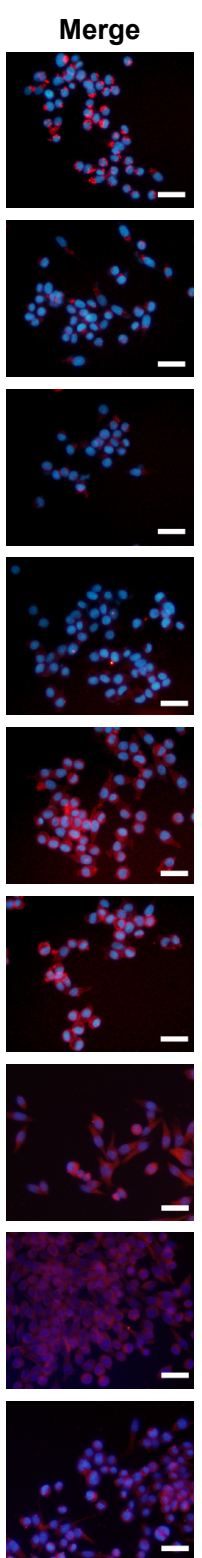

B
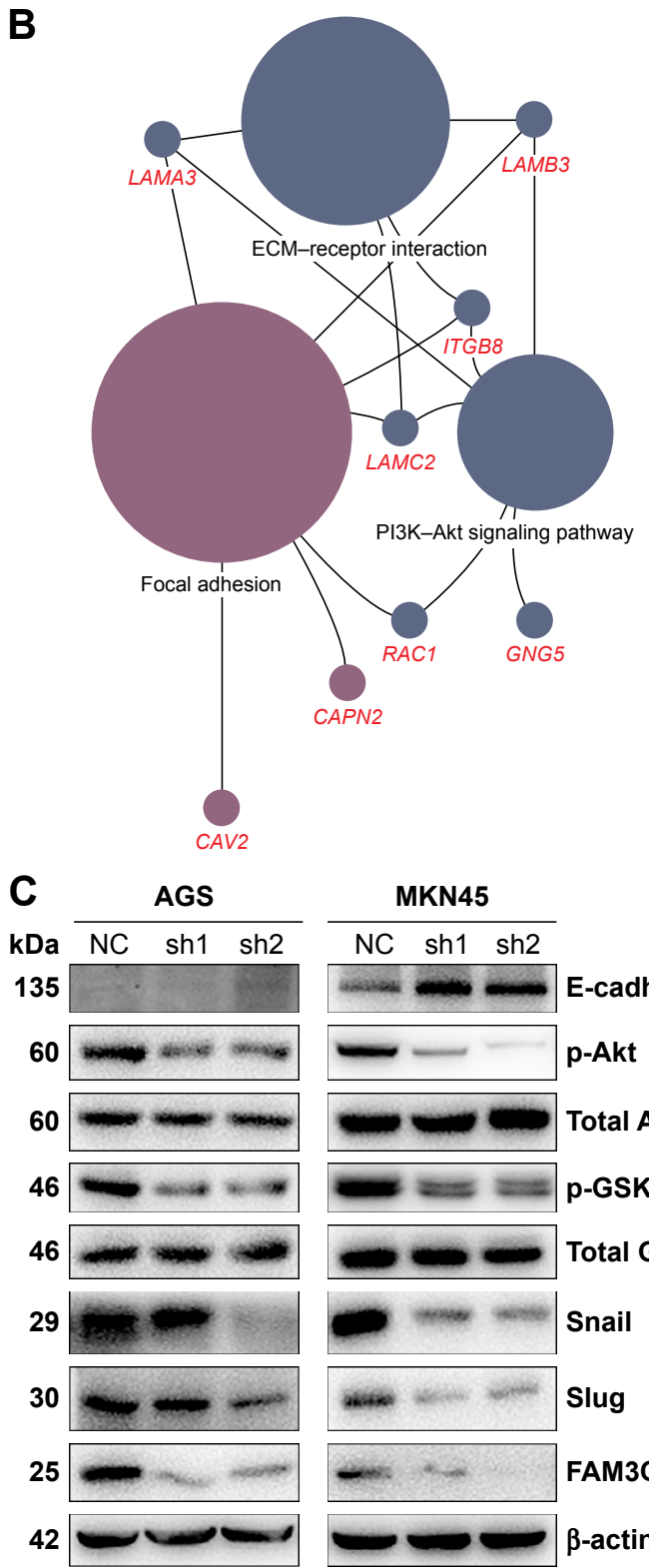

Figure 4 FAM3C reduction inhibited EMT and suppressed PI3K-Akt pathway activation in gastric cancer cells.

Notes: (A) Immunofluorescence of E-cadherin, vimentin, and FAM3C. E-cadherin was upregulated in FAM3C-knockdown MKN45 cells. The expression of vimentin in FAM3C-knockdown cells was decreased, as was mesenchymal cell morphology. Bar $50 \mu \mathrm{m}$. (B) Pattern diagram of the KEGG pathway enrichment of genes positively related to FAM $3 C$ in the gastric adenocarcinoma database of TCGA. Pathways with $P<0.05$ and $>5 \%$ associated genes are shown. Each circle represents a KEGG pathway term or a gene. (C) Western blotting showed that knockdown of FAM3C caused increased expression of E-cadherin and decreased levels of p-Akt, p-GSK3 $\beta$, Snail, and Slug (due to the mutation of E-cadherin in AGS cells, we could not detect E-cadherin by Western blotting in AGS cells).

Abbreviations: ECM, extracellular matrix; EMT, epithelial-mesenchymal transition; KEGG, Kyoto Encyclopedia of Genes and Genomes; NC, negative control; TCGA, the Cancer Genome Atlas.

Table I Enriched KEGG pathways of genes positively related to FAM3C in the gastric adenocarcinoma database of the Cancer Genome Atlas

\begin{tabular}{|l|l|l|l|l|}
\hline Term & Count & $\begin{array}{l}\text { Associated } \\
\text { genes (\%) }\end{array}$ & P-value & Genes \\
\hline Focal adhesion & 7 & 6.67 & 0.00017 & CAPN2, CAV2, ITGB8, LAMA3, LAMB3, LAMC2, RACI \\
\hline ECM-receptor interaction & 4 & 3.81 & 0.00556 & ITGB8, LAMA3, LAMB3, LAMC2 \\
\hline PI3K-Akt signaling pathway & 6 & 5.71 & 0.01330 & GNG5, ITGB8, LAMA3, LAMB3, LAMC2, RACI \\
\hline Pathways in cancer & 5 & 4.76 & 0.07958 & GNG5, LAMA3, LAMB3, LAMC2, RACI \\
\hline
\end{tabular}

Abbreviations: ECM, extracellular matrix; KEGG, Kyoto Encyclopedia of Genes and Genomes. 
A

HGC27
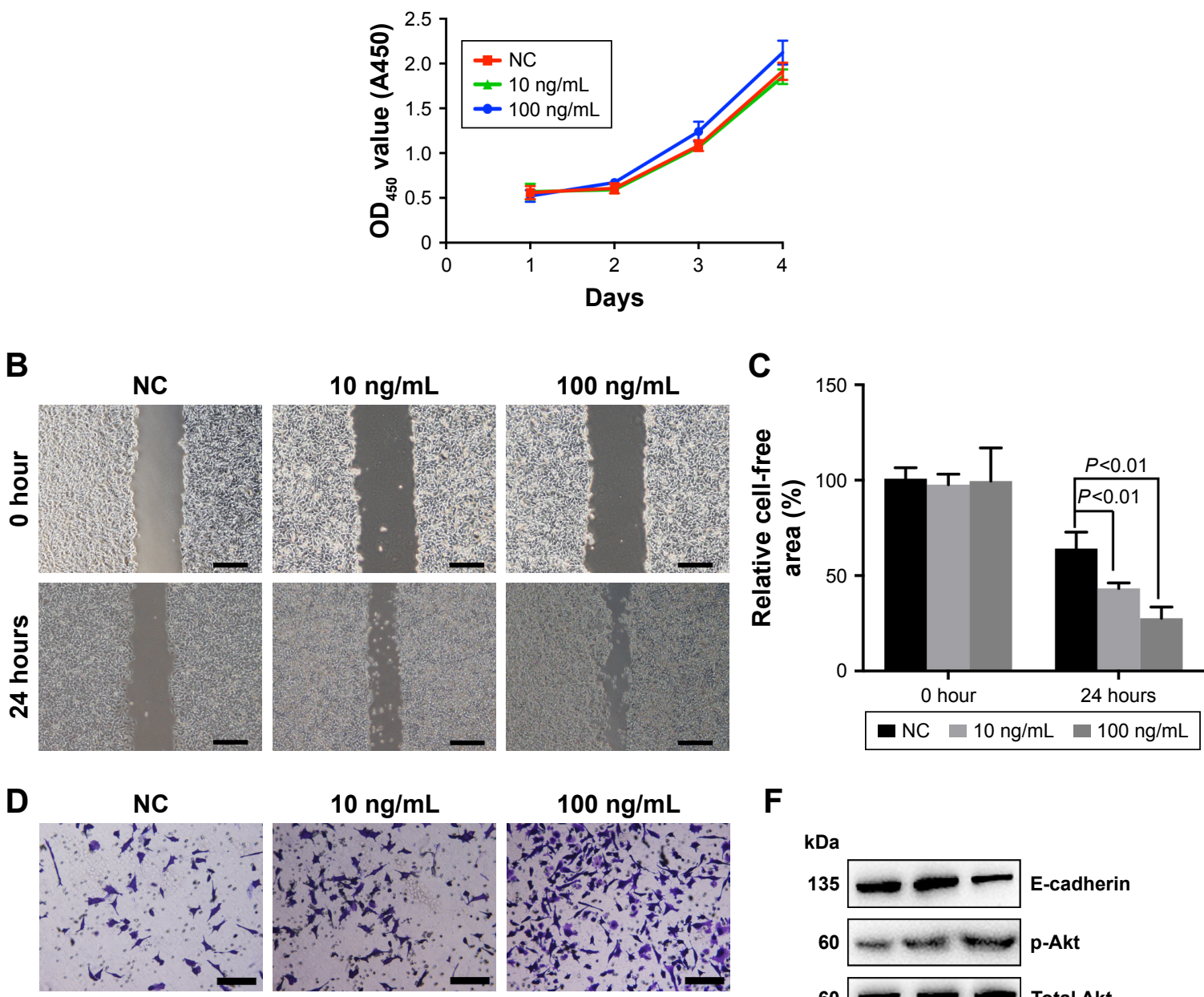

$\mathbf{F}$
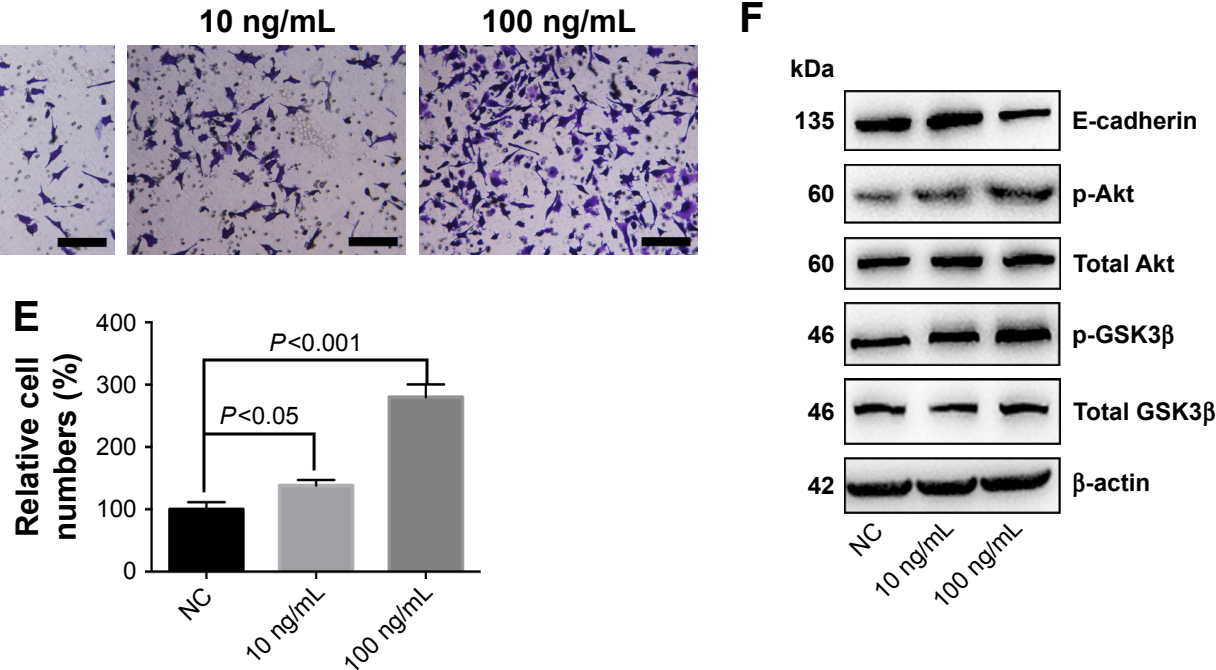

Figure 5 Exogenous FAM3C activated the PI3K/Akt pathway and promoted HGC27 cell migration.

Notes: (A) $\mathrm{OD}_{450}$ values of HGC27 cells treated with 0,10 and $100 \mathrm{ng} / \mathrm{mL}$ FAM3C at 24, 48, 72, and 96 hours after cells were seeded into 96 -well plates. (B, C) Exogenous FAM3C resulted in a smaller wound-healing area 24 hours after scratching. Representative images (bar $200 \mu \mathrm{m})(\mathbf{B})$ and statistics (C) regarding the percentage of uncovered area. (D, E) Exogenous FAM3C resulted in increased cell migration in the transwell assay 24 hours after cell seeding. Representative images (bar I00 $\mu \mathrm{m})(\mathbf{D})$ and statistics (E) regarding migrated cell numbers. (F) Western blot showing that exogenous FAM3C caused increased levels of p-Akt and p-GSK3 $\beta$ and decreased levels of E-cadherin. Abbreviation: NC, negative control.

mouse lateral tail veins, and five mice were injected in each group. The number of metastatic lesions on the largest section of each sample was calculated and the numbers analyzed 4 weeks after cell implantation. As shown in Figure 6A and B, knockdown of FAM3C significantly reduced the number of metastatic lesions.

\section{Discussion}

Secreted cytokine-activated RTKs play an important role in gastric cancer proliferation and metastasis. Autocrine cytokines and cytokines secreted by mesenchymal cells bind to a variety of RTKs and active downstream pathways, such as those involving PI3K/Akt, p-STAT3, and p-ERK. ${ }^{18,19}$ 


\section{A}

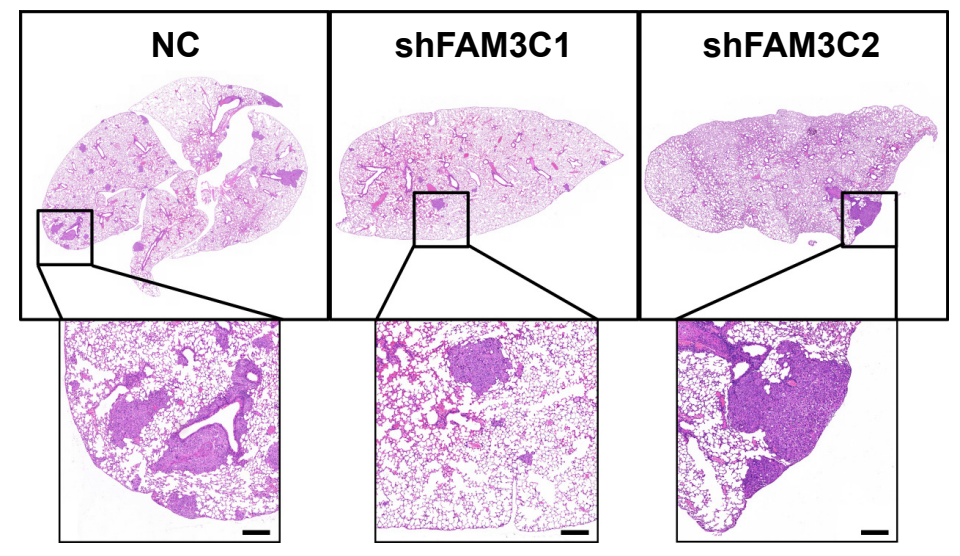

B

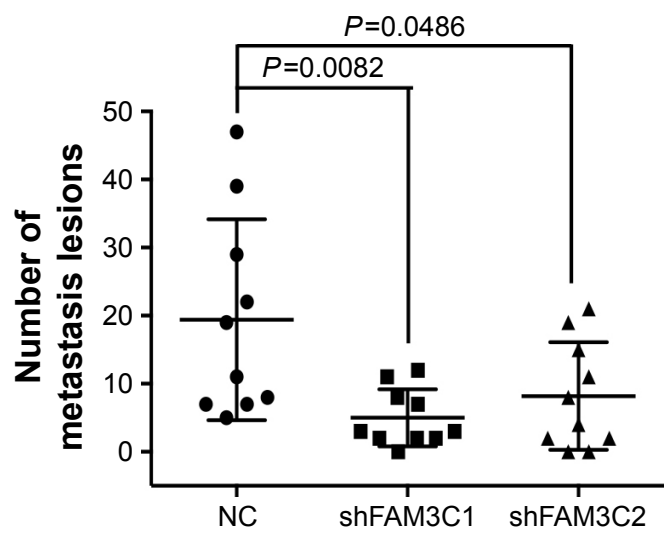

Figure 6 Knockdown of $F A M 3 C$ reduced gastric cancer metastatic lesions in vivo.

Notes: (A, B) Knockdown of FAM3C in MKN45 cells reduced the number of metastatic lesions in the largest section of each lung sample 4 weeks after cell injection. Representative H\&E staining of the largest lung section (bar $200 \mu \mathrm{m})(\mathbf{A})$ and statistics (B) regarding metastatic lesion numbers (B).

Abbreviation: NC, negative control.

Amplification or aberrant activation of RTKs resulting from genomic variation and amplification is also a driver of gastric cancer carcinogenesis and metastasis. The proportion of human epidermal growth factor receptor 2 (HER2)-positive gastric cancer ranges from $6 \%$ to $36.6 \% .^{20}$ In recent years, many tyrosine-kinase inhibitors and monoclonal antibodies targeting almost all kinds of RTKs have been invented and used in clinical trials. Trastuzumab, an antibody against HER2, and ramucirumab, an antibody against VEGFR2, have been approved by the US Food and Drug Administration to treat gastric cancer. These drugs have achieved some progress in gastric cancer treatment. An open-label international Phase III randomized controlled trial of trastuzumab in HER2-positive gastric cancer, referred to as ToGA, has been undertaken in 122 centers in 24 countries. The median overall survival time is 13.8 months $(95 \%$ CI $12-16)$ in patients assigned to trastuzumab plus chemotherapy compared with 11.1 months (95\% CI 10-13) in those assigned to chemotherapy alone (HR $0.74,95 \%$ CI $0.60-0.91 ; P=0.0046) .{ }^{21}$ However, progression, although valuable, is limited. A large proportion of gastric cancer patients relapse or do not respond during or after drug administration. Part of this might be ascribed to the fact that there are numerous kinds of cytokines targeting interacting receptors and that different downstream pathways often regulate interacting transcription factors. In addition, there is cross talk between those pathways.

Gastric cancer is similar to the hydra in Greek mythology: when one of its heads is removed, two grow back. The hero Heracles's methods of killing the Hydra, cauterizing the open stumps after cutting off each head, may inspire us to find a way to overcome gastric cancer. Elucidating every potential pathway and simultaneously inhibiting alternative pathways while blocking main pathways might be useful in the treatment of gastric cancer. TGF $\beta$ is a well-known cytokine that can activate EGFR and downstream ERK and PI3K-Akt pathways to promote gastric cancer metastasis. However, our study found that a novel cytokine, FAM3C, also promoted gastric cancer metastasis. When the TGF $\beta$ pathway is blocked, FAM3C might act as an alternative pathway to drive gastric cancer metastasis continuously.

EMT is the first step in epithelial cancer-cell metastasis. The canonical concept of EMT is that epithelial cells transform into mesenchymal cells. EMT is not only the first step in cancer cell metastasis but is also involved in several physiological and pathological processes, including embryonic development, tissue repair, and fibrosis. ${ }^{22}$ Cells undergoing EMT present certain features and gain an invasive phenotype. ${ }^{23}$ These features include disruption of intercellular junctions, loss of cell polarity, reorganization of the cytoskeleton, and increased cell motility. Therefore, in most experimental models, epithelial (E-cadherin) and mesenchymal (vimentin) markers and morphological changes are examined as indicators to confirm the occurrence of EMT. ${ }^{24}$

Regulation of cancer-cell EMT has received much attention. Various signaling pathways, such as those involving TGF $\beta$, Wnt, and growth-factor RTKs have been implicated in inducing EMT. ${ }^{25}$ TGF $\beta$ is considered a primary inducer of EMT. ${ }^{26}$ TGF $\beta$ deposited in surrounding stroma or secreted from tumor cells induces the expression of both ZEB1 and Snail, two EMT-related transcription factors, thereby triggering EMT to promote tumor progression and metastasis. ${ }^{27}$ Activation of growth-factor RTK pathways by either ligands 
or oncogenic mutation could also lead to EMT though downstream pathways. Inhibition of the PI3K-Akt-Snail pathway has been reported to attenuate EMT and suppress metastasis in lung cancer, breast cancer, gallbladder cancer, and gastric cancer. ${ }^{14-17}$

FAM3C, a novel cytokine, has been found to promote cancer-cell EMT and metastasis in several types of cancer, including breast cancer, colorectal cancer, bladder cancer, and lung cancer, either independently or in combination with TGF $\beta .^{5,28,29}$ Additionally, in gastric cancer, which was investigated by our study, overexpression of FAM3C existed and was related to worse prognosis, while knockdown of FAM3C attenuated EMT and metastasis of gastric cancer cells, both in vitro and in vivo. Moreover, exogenous FAM3C activated the PI3K-Akt pathway in HGC27 cells, which showed low FAM3C-expression levels, and caused enhanced cell migration. Notably, $100 \mathrm{ng} / \mathrm{mL}$ FAM3C also promotes HGC27-cell proliferation, which might be the result of PI3K-Akt pathway activation. ${ }^{30} \mathrm{KEGG}$ pathway enrichment of $F A M 3 C$-related genes in gastric cancer revealed enrichment in focal adhesion, extracellular matrix-receptor interaction, and $\mathrm{PI} 3 \mathrm{~K}$-Akt pathway categories. Western blot analysis showed that knockdown of FAM $3 C$ suppressed activation of the PI3K-Akt-GSK3 $\beta$ pathway and inhibited EMT in gastric cancer. FAM3C is among the nine heads of the hydra and might act as an alternative when other wellknown EMT-inducing cytokines are inhibited in gastric cancer. This possibility deserves further investigation, and should be taken into consideration during the development of new therapeutic strategies for gastric cancer.

\section{Conclusion}

The novel cytokine FAM3C participated in gastric cancer metastasis. Knockdown of FAM3C suppressed gastric cancer-cell migration and metastasis in vitro and in vivo. Reduced FAM3C attenuated activation of the PI3K-Akt signaling pathway. FAM3C should also be taken into consideration for gastric cancer treatments involving RTK pathways and cytokines.

\section{Acknowledgments}

This work was supported by the National Natural Science Foundation of China (81672935), Youth National Natural Science Foundation of China (81602147), Youth Natural Science Foundation of Jiangsu Province (BK20160110), Outstanding Youth Project of Nanjing City (JQX16026), a Nanjing international joint research project (201605082), Fundamental Research Funds for Central Universities
(021414380152), Nanjing Medical Science and Technique Development Foundation (QRX17037 and QRX17117), the Yunnan Provincial Innovation Group of Science and Technology (2018HC006).

\section{Author contributions}

$\mathrm{XZ}, \mathrm{SS}$, and MS were responsible for conception and design, MS and SN for acquisition of data, GD and MS for analysis and interpretation of data, MS for drafting of the article, and GD, SN, XZ, and SS for critical revision of the manuscript. All authors contributed toward data analysis, drafting and critically revising the paper, gave final approval of the version to be published, and agree to be accountable for all aspects of the work.

\section{Disclosure}

The authors report no conflicts of interest in this work.

\section{References}

1. van Cutsem E, Sagaert X, Topal B, Haustermans K, Prenen H. Gastric cancer. Lancet. 2016;388(10060):2654-2664.

2. Roviello G, Ravelli A, Polom K, et al. Apatinib: A novel receptor tyrosine kinase inhibitor for the treatment of gastric cancer. Cancer Lett. 2016;372(2):187-191.

3. Chang J, Wang S, Zhang Z, et al. Multiple receptor tyrosine kinase activation attenuates therapeutic efficacy of the fibroblast growth factor receptor 2 inhibitor AZD4547 in FGFR2 amplified gastric cancer. Oncotarget. 2015;6(4):2009-2022.

4. Zhu Y, Xu G, Patel A, et al. Cloning, expression, and initial characterization of a novel cytokine-like gene family. Genomics. 2002;80(2): $144-150$.

5. Waerner T, Alacakaptan M, Tamir I, et al. ILEI: a cytokine essential for EMT, tumor formation, and late events in metastasis in epithelial cells. Cancer Cell. 2006;10(3):227-239.

6. Gao ZH, Lu C, Wang ZN, et al. ILEI: a novel marker for epithelialmesenchymal transition and poor prognosis in colorectal cancer. Histopathology. 2014;65(4):527-538

7. Zhu YH, Zhang B, Li M, et al. Prognostic significance of FAM $3 \mathrm{C}$ in esophageal squamous cell carcinoma. Diagn Pathol. 2015;10:192.

8. Zhao X, Luo G, Fan Y, Ma X, Zhou J, Jiang H. ILEI is an important intermediate participating in the formation of TGF- $\beta 1$-induced renal tubular EMT. Cell Biochem Funct. 2018;36(2):46-55.

9. Chen Z, Ding L, Yang W, et al. Hepatic activation of the FAM3CHSF1-CaM pathway attenuates hyperglycemia of obese diabetic mice. Diabetes. 2017;66(5):1185-1197.

10. Duan G, Tang Q, Yan H, et al. A Strategy to delay the development of cisplatin resistance by maintaining a certain amount of cisplatinsensitive cells. Sci Rep. 2017;7(1):432.

11. Shannon P, Markiel A, Ozier O, et al. Cytoscape: a software environment for integrated models of biomolecular interaction networks. Genome Res. 2003;13(11):2498-2504.

12. Csiszar A, Kutay B, Wirth S, et al. Interleukin-like epithelial-tomesenchymal transition inducer activity is controlled by proteolytic processing and plasminogen-urokinase plasminogen activator receptor system-regulated secretion during breast cancer progression. Breast Cancer Res. 2014;16(5):433.

13. Wang LY, Liu YP, Chen LG, et al. Pyruvate kinase M2 plays a dual role on regulation of the EGF/EGFR signaling via E-cadherin-dependent manner in gastric cancer cells. PLoS One. 2013;8(6):e67542. 
14. Liu XL, Zhang XT, Meng J, et al. ING5 knockdown enhances migration and invasion of lung cancer cells by inducing EMT via EGFR/ PI3K/Akt and IL-6/STAT3 signaling pathways. Oncotarget. 2017; 8(33):54265-54276.

15. Wang M, Wu H, Li S, et al. SYNJ2BP promotes the degradation of PTEN through the lysosome-pathway and enhances breast tumor metastasis via PI3K/AKT/SNAI1 signaling. Oncotarget. 2017;8(52):89692-89706.

16. Li X, Teng S, Zhang Y, et al. TROP2 promotes proliferation, migration and metastasis of gallbladder cancer cells by regulating PI3K/AKT pathway and inducing EMT. Oncotarget. 2017;8(29):47052-47063.

17. Hao NB, Tang B, Wang GZ, et al. Hepatocyte growth factor (HGF) upregulates heparanase expression via the PI3K/Akt/NF- $\mathrm{KB}$ signaling pathway for gastric cancer metastasis. Cancer Lett. 2015;361(1) 57-66.

18. Achyut BR, Yang L. Transforming growth factor- $\beta$ in the gastrointestinal and hepatic tumor microenvironment. Gastroenterology. 2011; 141(4):1167-1178.

19. He Z, Dong W, Li Q, Qin C, Li Y. Sauchinone prevents TGF- $\beta$-induced EMT and metastasis in gastric cancer cells. Biomed Pharmacother. 2018;101:355-361.

20. Boku N. HER2-positive gastric cancer. Gastric Cancer. 2014;17(1): $1-12$.

21. Bang YJ, van Cutsem E, Feyereislova A, et al. Trastuzumab in combination with chemotherapy versus chemotherapy alone for treatment of HER2-positive advanced gastric or gastro-oesophageal junction cancer (ToGA): a phase 3, open-label, randomised controlled trial. Lancet. 2010;376(9742):687-697.
22. Stone RC, Pastar I, Ojeh N, et al. Epithelial-mesenchymal transition in tissue repair and fibrosis. Cell Tissue Res. 2016;365(3):495-506.

23. Davis FM, Stewart TA, Thompson EW, Monteith GR. Targeting EMT in cancer: opportunities for pharmacological intervention. Trends Pharmacol Sci. 2014;35(9):479-488.

24. Liao TT, Yang MH. Revisiting epithelial-mesenchymal transition in cancer metastasis: the connection between epithelial plasticity and stemness. Mol Oncol. 2017;11(7):792-804.

25. Yeung KT, Yang J. Epithelial-mesenchymal transition in tumor metastasis. Mol Oncol. 2017;11(1):28-39.

26. Katsuno Y, Lamouille S, Derynck R. TGF- $\beta$ signaling and epithelialmesenchymal transition in cancer progression. Curr Opin Oncol. 2013; 25(1):76-84

27. Korpal M, Lee ES, Hu G, Kang Y. The miR-200 family inhibits epithelial-mesenchymal transition and cancer cell migration by direct targeting of E-cadherin transcriptional repressors ZEB1 and ZEB2. J Biol Chem. 2008;283(22):14910-14914.

28. Song Q, Sheng W, Zhang X, Jiao S, Li F. ILEI drives epithelial to mesenchymal transition and metastatic progression in the lung cancer cell line A549. Tumour Biol. 2014;35(2):1377-1382.

29. Sun Y, Jia X, Gao Q, Liu X, Hou L. The ubiquitin ligase UBE4A inhibits prostate cancer progression by targeting interleukin-like EMT inducer (ILEI). IUBMB Life. 2017;69(1):16-21.

30. Osaki M, Oshimura M, Ito H. PI3K-Akt pathway: its functions and alterations in human cancer. Apoptosis. 2004;9(6):667-676. 


\section{Supplementary material}

Table SI Genes positively related to FAM3C from TCGA gastric cancer data

\begin{tabular}{|c|c|c|c|}
\hline Gene symbol & Cytoband & $\begin{array}{l}\text { Pearson } \\
\text { score }\end{array}$ & $\begin{array}{l}\text { Spearman } \\
\text { score }\end{array}$ \\
\hline REEP3 & $10 q 21.3$ & 0.3 & 0.39 \\
\hline PAXIPI-AS2 & $7 q 36.2$ & 0.3 & 0.31 \\
\hline CHMP2B & $3 p \mid 1.2$ & 0.3 & 0.34 \\
\hline SNORA8 & I Iq2I & 0.3 & 0.33 \\
\hline FLJ44635 & $X q \mid 3.1$ & 0.3 & 0.27 \\
\hline $\mathrm{HRHI}$ & $3 p 25.3$ & 0.31 & 0.28 \\
\hline THAP5 & $7 q 31.1$ & 0.31 & 0.38 \\
\hline MTPN & $7 q 33$ & 0.31 & 0.38 \\
\hline RACl & $7 p 22.1$ & 0.31 & 0.39 \\
\hline RASAI & $5 q \mid 4.3$ & 0.31 & 0.3 \\
\hline CI8ORF32 & $18 q 21.1$ & 0.31 & 0.33 \\
\hline SYPLI & $7 q 22.3$ & 0.31 & 0.39 \\
\hline PHLDA2 & IIpI5.4 & 0.31 & 0.32 \\
\hline CCDCI 75 & I4q23.1 & 0.31 & 0.32 \\
\hline NEK7 & $\mid q 31.3$ & 0.31 & 0.31 \\
\hline SPCS2 & ||$q \mid 3.4$ & 0.31 & 0.28 \\
\hline UBE2E3 & $2 q 31.3$ & 0.31 & 0.31 \\
\hline GULPI & $2 q 32.1-q 32.2$ & 0.31 & 0.21 \\
\hline BTBD IO & IIpI5.3 & 0.31 & 0.33 \\
\hline TMEMI85A & Xq28 & 0.31 & 0.21 \\
\hline TXNDCI 7 & $17 p \mid 3.1$ & 0.31 & 0.35 \\
\hline OCIADI & $4 p I I$ & 0.31 & 0.37 \\
\hline UBA6 & $4 q 13.2$ & 0.31 & 0.37 \\
\hline APIAR & $4 q 25$ & 0.31 & 0.34 \\
\hline SLC25A40 & $7 q 21.12$ & 0.31 & 0.38 \\
\hline SECI 4L2 & $22 q \mid 2.2$ & 0.31 & 0.15 \\
\hline CIGALTI & $7 p 22.1-p 21.3$ & 0.31 & 0.38 \\
\hline IFT80 & $3 q 25.33$ & 0.31 & 0.42 \\
\hline OSBPL3 & $7 p 15.3$ & 0.31 & 0.34 \\
\hline CLVSI & $8 q|2.2-q| 2.3$ & 0.31 & 0.36 \\
\hline TINAGLI & Ip35.2 & 0.31 & 0.08 \\
\hline LRRCI9 & $9 p 21.2$ & 0.31 & 0.19 \\
\hline TMEM/35 & ||$q \mid 4.2$ & 0.31 & 0.38 \\
\hline CBFB & 16q22.1 & 0.32 & 0.39 \\
\hline LAMA3 & $|8 q| \mid .2$ & 0.32 & 0.29 \\
\hline LAMC2 & Iq25.3 & 0.32 & 0.28 \\
\hline SCP2 & Ip32.3 & 0.32 & 0.38 \\
\hline UBE2D I & $10 \mathrm{q} 21.1$ & 0.32 & 0.41 \\
\hline SNORD $/ 23$ & $5 p 15.31$ & 0.32 & 0.26 \\
\hline SREKI & $5 q \mid 2.3$ & 0.32 & 0.37 \\
\hline TMEM4 I B & IIpI5.4 & 0.32 & 0.38 \\
\hline TM4SF20 & $2 q 36.3$ & 0.32 & 0.2 \\
\hline INSIGZ2 & $2 q|4 . I-q| 4.2$ & 0.32 & 0.39 \\
\hline
\end{tabular}

Table SI (Continued)

\begin{tabular}{|c|c|c|c|}
\hline Gene symbol & Cytoband & $\begin{array}{l}\text { Pearson } \\
\text { score }\end{array}$ & $\begin{array}{l}\text { Spearman } \\
\text { score }\end{array}$ \\
\hline MRPS33 & $7 q 34$ & 0.32 & 0.35 \\
\hline TMEM / 28 & $4 p \mid 6.3$ & 0.32 & 0.37 \\
\hline GSAP & $7 q 11.23$ & 0.32 & 0.33 \\
\hline EQTN & $9 p 21.2$ & 0.32 & 0.33 \\
\hline ZNF654 & $3 p 11.1$ & 0.32 & 0.32 \\
\hline$P I 4 K 2 B$ & $4 p \mid 5.2$ & 0.32 & 0.33 \\
\hline TLDCI & $16 q 24.1$ & 0.32 & 0.29 \\
\hline RSBNIL & $7 q 11.23$ & 0.32 & 0.3 \\
\hline$D D \times 60 L$ & $4 q 32.3$ & 0.32 & 0.34 \\
\hline TMEMI 70A & $16 q 23.1$ & 0.32 & 0.39 \\
\hline SLC25A24 & $|p| 3.3$ & 0.32 & $0.4 I$ \\
\hline TORIAIP2 & $\operatorname{lq} 25.2$ & 0.32 & 0.37 \\
\hline NT5E & $6 q \mid 4.3$ & 0.33 & 0.11 \\
\hline ZNF800 & $7 q 31.33$ & 0.33 & 0.37 \\
\hline ARHGEF5 & $7 q 35$ & 0.33 & 0.33 \\
\hline$B L Z F I$ & Iq24.2 & 0.33 & 0.34 \\
\hline TCAFI & $7 q 35$ & 0.33 & 0.37 \\
\hline RAD50 & $5 q 31.1$ & 0.33 & 0.28 \\
\hline CAAPI & $9 p 21.2$ & 0.33 & 0.29 \\
\hline SFT2DI & $6 q 27$ & 0.33 & 0.31 \\
\hline TMEM/33 & I Iq22.I & 0.33 & 0.34 \\
\hline MIR4260 & $1 \mathrm{q} 32.2$ & 0.33 & 0.21 \\
\hline LOCI00I30705 & $7 q 32.1$ & 0.33 & 0.28 \\
\hline LSM5 & $7 p \mid 4.3$ & 0.33 & 0.34 \\
\hline GRAMD2B & $5 q 23.2$ & 0.34 & 0.38 \\
\hline ITGB8 & $7 p 21.1$ & 0.34 & 0.38 \\
\hline LAMB3 & Iq32.2 & 0.34 & 0.22 \\
\hline$B C L I O$ & Ip22.3 & 0.34 & 0.38 \\
\hline LAMTOR5 & $|p| 3.3$ & 0.34 & 0.39 \\
\hline TMEM60 & $7 q 11.23$ & 0.34 & 0.33 \\
\hline SMIMI5 & $5 q \mid 2.1$ & 0.34 & 0.34 \\
\hline EFCAB IO & $7 q 22.3$ & 0.34 & 0.5 \\
\hline NAPEPLD & $7 q 22.1$ & 0.34 & 0.47 \\
\hline$A L S 2 C L$ & $3 p 21.31$ & 0.34 & 0.31 \\
\hline FAM IIIA & $I|q| 2.1$ & 0.34 & 0.36 \\
\hline DENNDIB & Iq31.3 & 0.34 & 0.35 \\
\hline FDXI & I Iq22.3 & 0.35 & 0.26 \\
\hline LAMTOR3 & $4 q 23$ & 0.35 & 0.41 \\
\hline TANK & $2 q 24.2$ & 0.35 & 0.46 \\
\hline IFNE & $9 p 21.3$ & 0.35 & 0.26 \\
\hline SLC37A3 & $7 q 34$ & 0.35 & 0.37 \\
\hline ING3 & $7 q 31.31$ & 0.35 & 0.32 \\
\hline GORAB & Iq24.2 & 0.35 & 0.41 \\
\hline CAV2 & $7 q 31.2$ & 0.36 & 0.24 \\
\hline OCIAD2 & $4 \mathrm{pll}$ & 0.36 & 0.41 \\
\hline SLC20AI & $2 q 14.1$ & 0.36 & 0.22 \\
\hline
\end{tabular}


Table SI (Continued)

\begin{tabular}{|c|c|c|c|}
\hline Gene symbol & Cytoband & $\begin{array}{l}\text { Pearson } \\
\text { score }\end{array}$ & $\begin{array}{l}\text { Spearman } \\
\text { score }\end{array}$ \\
\hline TMEM243 & $7 q 21.12$ & 0.36 & 0.4 \\
\hline SLC30A7 & $|p 2| .2$ & 0.36 & 0.45 \\
\hline TMEMIO6B & $7 p 21.3$ & 0.36 & 0.36 \\
\hline CAPN2 & Iq4I & 0.37 & 0.35 \\
\hline GNG5 & Ip22.3 & 0.37 & 0.39 \\
\hline MIR3IHG & $9 p 21.3$ & 0.37 & 0.23 \\
\hline APIS3 & $2 q 36.1$ & 0.37 & 0.38 \\
\hline PNPLA8 & $7 q 31.1$ & 0.38 & 0.43 \\
\hline LSM8 & $7 q 31.31$ & 0.38 & 0.46 \\
\hline STMPI & $7 q 33$ & 0.38 & 0.41 \\
\hline CTBS & Ip22.3 & 0.39 & 0.43 \\
\hline ARHGEF35 & $7 q 35$ & 0.39 & 0.38 \\
\hline CD58 & $|p| 3.1$ & 0.4 & 0.45 \\
\hline CMTR2 & $16 q 22.2$ & 0.4 & 0.44 \\
\hline ANXA2P3 & $10 q 21.3$ & 0.42 & 0.43 \\
\hline PTPN/2 & $7 q 11.23$ & 0.42 & 0.43 \\
\hline PARD6B & $20 q 13.13$ & 0.44 & 0.4 \\
\hline
\end{tabular}

Abbreviation: TCGA, the Cancer Genome Atlas.

\section{Publish your work in this journal}

OncoTargets and Therapy is an international, peer-reviewed, open access journal focusing on the pathological basis of all cancers, potential targets for therapy and treatment protocols employed to improve the management of cancer patients. The journal also focuses on the impact of management programs and new therapeutic agents and protocols on

\section{Dovepress}

patient perspectives such as quality of life, adherence and satisfaction. The manuscript management system is completely online and includes a very quick and fair peer-review system, which is all easy to use. Visit http://www.dovepress.com/testimonials.php to read real quotes from published authors. 